\title{
The Impact of Previous Ureteroscopic Tumor Ablation on Oncologic Outcomes After Radical Nephrouretectomy for Upper Urinary Tract Urothelial Carcinoma
}

\author{
Cenk Gurbuz, M.D., ${ }^{1, *}$ Ramy F. Youssef, M.D., ${ }^{1, *}$ Shahrokh F. Shariat, M.D., ${ }^{2}$ Yair Lotan, M.D., ${ }^{1}$ \\ Christopher G. Wood, M.D., ${ }^{3}$ Arthur I. Sagalowsky, M.D., Richard Zigeuner, M.D., ${ }^{4}$ Eiji Kikuchi, M.D., \\ Alon Weizer, M.D., ${ }^{6}$ Jay D. Raman, M.D., ${ }^{7}$ Mesut Remzi, M.D., ${ }^{8}$ Marco Roscigno, M.D., ${ }^{9}$ \\ Francesco Montorsi, M.D., ${ }^{9}$ Christian Bolenz, M.D., ${ }^{10}$ Wassim Kassouf, M.D.," and Vitaly Margulis, M.D.
}

\begin{abstract}
We investigated whether a history of endoscopic tumor ablation impacts oncologic outcomes after radical nephroureterectomy (RNU) for upper urinary tract urothelial carcinoma (UTUC). Using a multi-institutional database that contained patients who were treated with RNU, oncologic outcomes were assessed according to history of ureteroscopic tumor ablation. Disease-free survival (DFS) and cancer-specific survival (CSS) were estimated using the Kaplan-Meier survival analysis. Multivariate Cox regression analyses were performed to determine independent predictors of disease recurrence and cancer-specific mortality after RNU. The study included 1268 patients, 853 men and 415 women, with a mean age of 67.5 years (range 32-94 y) and 52.8 months median follow-up after RNU. A total of 175 (13\%) patients underwent RNU after endoscopic tumor ablation and $1093(87 \%)$ patients underwent RNU without a history of endoscopic ablation. The 5-year DFS and CSS rates were $72 \%$ and $77 \%$ in those with a history of tumor ablation $v s 69 \%$ and $73 \%$ in those without a history of ablation $(P=0.171$ and $P=0.365$, respectively). In multivariate Cox regression analysis, history of ablation therapy was not associated with disease recurrence or cancer-specific mortality (hazard ratio [HR]: 0.79, $P=0.185$ and HR: 0.7, $P=0.078$, respectively). Our collaborative international efforts suggest that in selected patients, endoscopic tumor ablation does not adversely affect the recurrence and survival after subsequent RNU for UTUC. Our data support the continued role of ureteroscopic ablation of UTUC in appropriately selected patients.
\end{abstract}

\section{Introduction}

$\mathbf{U}$ PPER URINARY TRACT UROTHELIAL CARCINOMA (UTUC) is relatively rare, accounting for approximately $5 \%$ of genitourinary malignancies, although recent data suggest that the incidence is increasing. ${ }^{1,2}$ The standard treatment for most patients with localized UTUC has been radical nephroureterectomy (RNU). ${ }^{2}$

Advances in flexible fiberoptic instruments and improvements in laser technology have allowed for organ-sparing endoscopic management of low-grade, low-stage UTUC. Conflicting data exist, however, regarding the oncologic safety of endoscopic interventions for UTUC, with theoretical concerns centering on inadequate clinical tumor staging, incomplete ablation, and increased risk of tumor dissemination. ${ }^{3,4}$ In addition, the impact of delay to RNU in patients with UTUC because of an attempt at ablative therapy is not well characterized. ${ }^{5}$ Similar to the reported experience in bladder cancer, attempts at an endoscopic intervention before RNU may result in a delay to definitive surgical therapy with

\footnotetext{
${ }^{1}$ University of Texas Southwestern Medical Center, Dallas, Texas.

${ }^{2}$ New York Presbyterian Hospital, Weill Medical College of Cornell University, New York, New York.

${ }^{3}$ University of Texas M.D. Anderson Cancer Center, Houston, Texas.

${ }^{4}$ Medical University of Graz, Graz, Austria.

${ }^{5}$ Keio University School of Medicine, Tokyo, Japan.

${ }^{6}$ University of Michigan, Ann Arbor, Michigan.

${ }^{7}$ Division of Urology, Penn State Milton S. Hershey Medical Center, Hershey, Pennsylvania.

${ }^{8}$ Medical University of Vienna, Vienna, Austria.

${ }^{9}$ Vita-Salute University, Milan, Italy.

${ }^{10}$ Mannheim Medical Center, University of Heidelberg, Mannheim, Germany.

${ }^{11}$ McGill University, Montreal, Quebec, Canada.

${ }^{*}$ Cenk Gurbuz and Ramy F. Youssef contributed equally.
} 
increased risks of disease progression and adverse impact on oncologic outcome. ${ }^{6,7}$ Addressing these concerns, we sought to retrospectively review outcomes of patients with UTUC who were treated with endoscopic ablation before RNU compared with those solely treated by RNU.

\section{Patients and Methods}

\section{Patient selection}

We used a database that comprised patients from the UTUC Collaboration; this patient cohort has previously been described in the literature. ${ }^{8}$ This was a study approved by an Institutional Review Board, with all participating sites providing the necessary institutional data use agreements before initiation of the study. After combining the data sets from the enrolled centers, a database containing 1363 patients who underwent RNU between 1987 and 2007 was generated. Excluding patients with a history of radical cystectomy and patients who were treated with neoadjuvant chemotherapy before RNU, the final dataset comprised 1268 patients who were treated with RNU for UTUC. Patients were evaluated before ureteroscopic ablation and/or RNU by cystoscopy, retrograde ureteropyelography, ureteroscopy, and cytology / biopsy. Staging radiologic imaging included intravenous urography (IVU), ultrasonography, and CT of the abdomen and pelvis. Diagnostic and staging criteria varied significantly during the time of the study and among participating centers.

Although selection criteria for ureteroscopic ablation varied among participating institutions, generally endoscopic tumor ablation was offered electively to patients with completely resected, low-grade, unifocal, noninvasive tumors. Ureteroscopic ablation was also offered to selected patients with high-grade bilateral tumors, renal insufficiency, tumors in a solitary renal unit, and to persons not medically fit for RNU. Endoscopic tumor ablation was defined as electrocautery or laser-based destruction of all grossly visible disease within the upper urinary tract. ${ }^{9,10}$ RNU was performed because of tumor recurrence/persistence, not amenable to repeated ablation, evidence of stage or grade progression, and patient or clinician preference.

\section{Pathologic evaluation}

All surgical specimens were processed according to standard pathologic procedures, and all slides were rereviewed by genitourinary pathologists at participating centers according to prospectively defined uniform criteria. All pathologists were blinded to clinical outcomes. Tumors were staged according to the sixth edition of the American Joint Committee on Cancer-Union International Contre le Cancer Tumor-Node-Metastasis classification. ${ }^{11}$ Tumor grades were assessed according to the 1998 World Health Organization/ International Society of Urologic Pathology consensus classification. $^{12}$

\section{Surveillance regimen}

Follow-up was performed according to institutional protocols. Patients were generally followed every 3 months after endoscopic ablation. The follow-up included cystocopy, ureteroscopy, and cytology. Imaging of the upper tract by IVU or CT was performed every 6 to 12 months. After RNU, patients were followed every 3 months for the first year and every 6 months thereafter. Follow-up consisted of a history, physical examination, routine blood work and serum chemistry studies, urinary cytology, chest radiography, cystoscopic evaluation of the urinary bladder, and radiographic evaluation of the contralateral upper urinary tract. Elective bone scans, chest CT, or MRI were performed when indicated clinically.

\section{Outcome assessment and statistical analysis}

Bladder recurrences were not considered in the analysis of disease-free survival (DFS) rate. DFS and cancer-specific survival (CSS) were estimated using the Kaplan-Meier survival analysis. Disease recurrence was defined as local failure in the nephroureterectomy bed, regional lymph nodes, or distant metastasis after RNU for UTUC. The period of DFS was defined as the time between the date of RNU and the development of local recurrence or distant metastasis. Censored survival values represent patients who were alive without clinical evidence of disease at the last follow-up. Cause of death was determined by the treating physicians, by chart review corroborated by death certificates, or by death certificates alone. The period of CSS was defined as the time between the date of RNU and death from cancer. Statistical differences were determined by the log rank test. All reported $P$ values are two-sided, and significance was set at 0.05 . Finally, multivariate Cox regression analysis was performed to determine the independent predictors of disease recurrence and cancer-specific mortality after RNU. All statistical tests were performed with SPSS version 17.0.

\section{Results}

The clinical characteristics of the study population and final pathologic findings from RNU specimens are presented in Table 1 . The cohort of 1268 patients who underwent RNU included 853 men and 415 women with a median age of 68.5 years (range 32-94 y). Median follow-up for the whole cohort was 52.8 months (range 3-250 mos), with a total of 175 patients undergoing RNU after endoscopic tumor ablation and 1093 patients treated with RNU without a history of tumor ablation. Adjuvant systemic chemotherapy was administered to $25(14.4 \%)$ and $128(11.7 \%)$ patients after RNU in the initial ablation and initial RNU cohorts, respectively $(P=0.318)$.

UTUC recurred in $36(20 \%)$ patients who were treated with initial tumor ablation and in $303(28 \%)$ patients who were treated with initial RNU. Cancer-specific death was documented in $30(17 \%)$ patients who were treated with initial tumor ablation and in $250(23 \%)$ patients who were treated with initial RNU. Kaplan-Meier survival analyses demonstrated similar recurrence and survival rates in patients after RNU, regardless of history of endoscopic tumor ablation (Fig. 1). The 5-year DFS estimates were $72 \%$ and $69 \%$ in initial ablation and initial RNU patients, respectively $(P=0.171)$. The 5-year CSS estimates were $77 \%$ and $73 \%$ in initial ablation and initial RNU patients, respectively $(P=0.365)$.

In multivariate Cox regression analyses, tumor stage, grade, architecture, and lymph node involvement were independent predictors of DFS and CSS. History of endoscopic tumor ablation, however, was not associated with disease recurrence or cancer-specific mortality after RNU (hazard ratio [HR]: 0.79 , confidence interval $[\mathrm{CI}]: 0.55-1.12, P=0.185$ and HR: 0.70, CI: 0.48-1.04, $P=0.078$, respectively) (Table 2). 
Table 1. The Clinical And Pathologic Characteristics Of Patients With Upper Urinary Tract Urothelial Carcinoma According to Initial Management Modality

\begin{tabular}{|c|c|c|c|c|}
\hline & \multirow[b]{2}{*}{ Entire cohort } & \multicolumn{2}{|c|}{ Initial management } & \multirow[b]{2}{*}{ P value } \\
\hline & & Ablation (175) & RNU (1093) & \\
\hline \multirow{2}{*}{$\begin{array}{l}\text { Age }(y) \\
\text { (mean, median and range) }\end{array}$} & & & & \multirow[t]{2}{*}{0.011} \\
\hline & $\begin{array}{c}68.3,69.6 \\
(32-94)\end{array}$ & $\begin{array}{c}70.3,71.2 \\
(40-94)\end{array}$ & $\begin{array}{c}68.1,69.2 \\
(32-89)\end{array}$ & \\
\hline \multicolumn{4}{|l|}{ Sex $(\%)$} & \multirow[t]{3}{*}{0.030} \\
\hline Female & $415(33 \%)$ & $45(25 \%)$ & $370(34 \%)$ & \\
\hline Male & $853(67 \%)$ & $130(75 \%)$ & $723(66 \%)$ & \\
\hline \multicolumn{4}{|l|}{ Tumor location (\%) } & \multirow{3}{*}{0.067} \\
\hline Kidney & $838(66 \%)$ & $105(60 \%)$ & $733(67 \%)$ & \\
\hline Ureter & $430(34 \%)$ & $70(40 \%)$ & $360(33 \%)$ & \\
\hline \multicolumn{4}{|l|}{ RNU (\%) } & \multirow{3}{*}{0.010} \\
\hline Open & $970(76 \%)$ & $88(50 \%)$ & $882(81 \%)$ & \\
\hline Laparoscopic & $298(24 \%)$ & $87(50 \%)$ & $211(19 \%)$ & \\
\hline Adjuvant chemotherapy (\%) & $153(12 \%)$ & $25(14 \%)$ & $128(11 \%)$ & 0.318 \\
\hline \multicolumn{4}{|l|}{ Pathologic tumor stage (\%) } & \multirow{7}{*}{$<0.01$} \\
\hline $\mathrm{T}_{\mathrm{a}}$ & $259(20 \%)$ & $73(42)$ & $186(17 \%)$ & \\
\hline $\mathrm{T}_{\mathrm{is}}$ & $22(1 \%)$ & $6(3)$ & $16(1 \%)$ & \\
\hline $\mathrm{T}_{1}$ & $286(23 \%)$ & $41(23 \%)$ & $245(22 \%)$ & \\
\hline $\mathrm{T}_{2}$ & $234(19 \%)$ & $36(21 \%)$ & $216(20 \%)$ & \\
\hline $\mathrm{T}_{3}$ & $408(32 \%)$ & $18(10.5 \%)$ & $372(35 \%)$ & \\
\hline $\mathrm{T}_{4}$ & $59(5 \%)$ & $1(0.5 \%)$ & $58(5 \%)$ & \\
\hline \multicolumn{4}{|l|}{ Pathologic grade $(\%)$} & \multirow{3}{*}{0.627} \\
\hline Low & $479(38 \%)$ & $69(40 \%)$ & $410(38 \%)$ & \\
\hline High & $789(62 \%)$ & $106(60 \%)$ & $683(62 \%)$ & \\
\hline \multicolumn{4}{|l|}{ Tumor architecture (\%) } & \multirow{3}{*}{0.612} \\
\hline Papillary & $944(74 \%)$ & $133(76 \%)$ & $811(74 \%)$ & \\
\hline Sesile & $324(26 \%)$ & $42(24 \%)$ & $282(26 \%)$ & \\
\hline Lymphovascular invasion & $297(23 \%)$ & $22(13 \%)$ & $275(25 \%)$ & 0.010 \\
\hline Lymph node involvement & $116(9 \%)$ & $6(3 \%)$ & $110(10 \%)$ & 0.050 \\
\hline
\end{tabular}

$\mathrm{RNU}=$ radical nephroureterectomy.

\section{Discussion}

It has been reported that urothelial carcinoma behaves identically in the upper and lower urinary tracts when stage and grade are matched. ${ }^{13}$ Because of our ability to successfully manage appropriately selected patients with bladder cancer cystoscopically, endoscopic management of UTUC is an attractive option, potentially obviating the need for a major extirpative surgery and allowing maximal preservation of renal function. Although advanced endourologic technologies that are applicable to management of UTUC are currently available, lacking appropriate clinical trials, concerns regarding oncologic safety of their application remain. Unlike in bladder cancer, accurate clinical staging and monitoring of UTUC is difficult, with significant rates of upstaging and upgrading at RNU. ${ }^{14}$ Furthermore, in parallel to the bladder cancer literature, delay to RNU attributable to understaging and inappropriate endoscopic management of UTUC may negatively impact oncologic outcomes. ${ }^{6,7}$ Finally, there are theoretical concerns about tumor dissemination via urothelial perforation, pyelolymphatic, or pyelovenous backflow. ${ }^{3,4}$ In addition, the effectiveness of topical immunotherapy or chemotherapy for adjuvant treatment of upper tract urothelial tumors is unclear.

Unfortunately, there are little data that evaluate the impact of initial endoscopic tumor ablation on survival outcomes after RNU for UTUC. In one of the few studies that evaluate the impact of delay to RNU in patients undergoing initial endoscopic tumor ablation, Boorjian and collaborators ${ }^{5}$ concluded that endoscopic ablation before RNU did not adversely affect the postoperative oncologic outcomes. Conclusions from this study, however, may be limited by the fact that only 12 patients in their cohort underwent RNU after ureteroscopic ablation. We present one of the largest clinical experiences in UTUC, with a significant number of patients $(175[13 \%])$ who were treated with initial endoscopic tumor ablation before radical surgery. As expected, patients initially selected for RNU were more likely to have features that were associated with advanced UTUC, such as higher tumor stage, presence of lymphovascular invasion, and increased incidence of regional lymphatic metastases, compared with patients who were initially selected for endoscopic management. In univariate and multivariate analysis that adjusted for these factors, however, history of ureteroscopic ablation was not associated with inferior DFS or CSS.

Laparoscopic RNU was performed more frequently in the initial ablation group compared with the initial RNU group (50\% vs $20 \%, P=0.01$ ), suggesting the applicability of this approach in the majority of UTUC patients in whom initial endoscopic treatment fails. We also noted a higher probability of ureteroscopic ablation in elderly patients. The median age of patients who underwent initial ablation was 71 years (range 40-94 y), and it was significantly higher than patients 


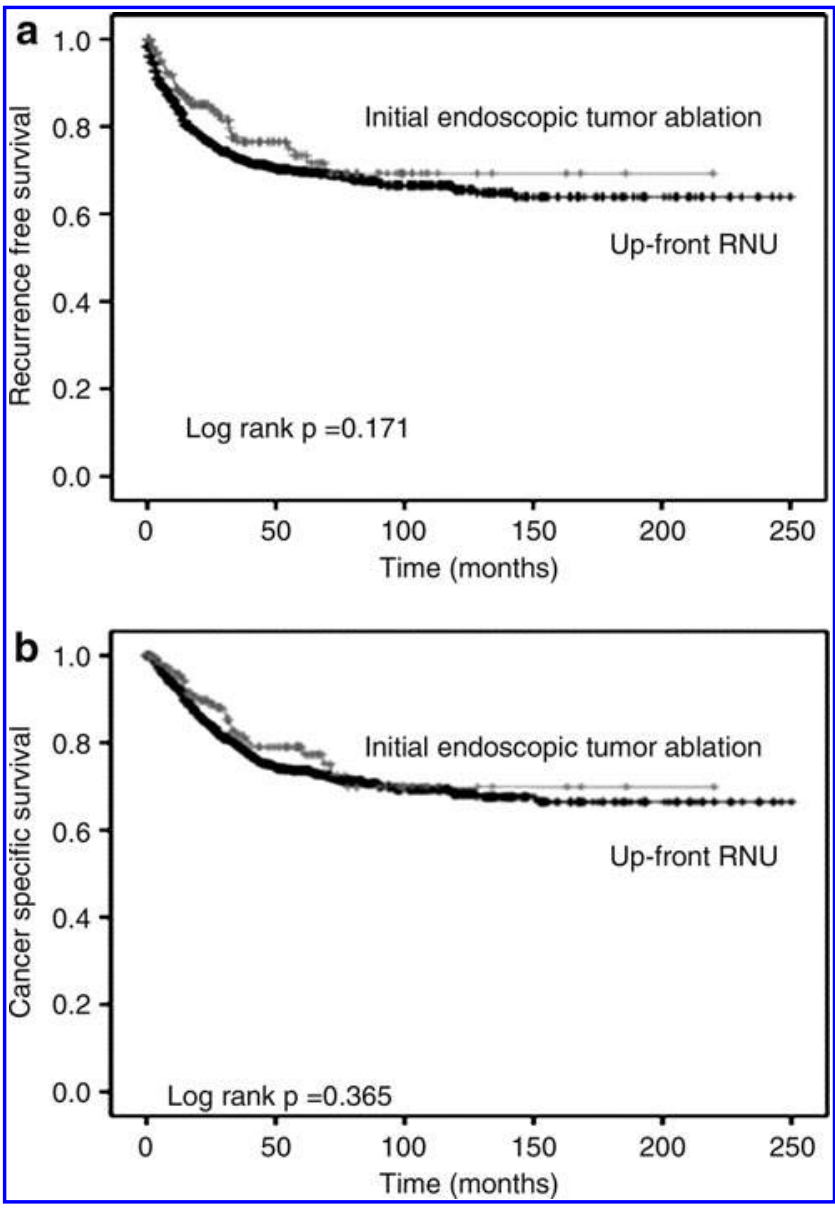

FIG. 1. Kaplan-Meier estimates for (a) recurrence-free survival and (b) cancer-specific survival in 1268 patients treated by radical nephroureterectomy (RNU) for upper urinary tract urothelial carcinoma according to history of endoscopic tumor ablation.

who underwent immediate RNU, whose median age was 69 years (range 32-89 y). The age-related comorbidites and the concerns about declining global renal function may explain why initial ablation was offered to older patients.
While endoscopic tumor ablation remains a viable option for selected patients with UTUC, tools to improve clinical staging and risk stratification of upper tract disease are urgently needed to optimize care of these patients. Various clinical variables, such as biopsy grade, ipsilateral hydonephropsis, and urine cytology have been correlated with pathologic findings at RNU, but individual variable predictive accuracy remains low. ${ }^{14,15}$ Using a multivariable outcome model, we have recently developed a preoperative nomogram, based on grade, architecture, and location of the tumor to predict the probability of nonorgan-confined UTUC with improved accuracy. ${ }^{16}$ Ultimately, multivariable models that incorporate molecular tumor markers will further improve our ability to accurately predict clinical tumor behavior.

The current study is not devoid of limitations. First, the power of our conclusions is weakened by the retrospective nature of the study, with inherent limitations attributable to this study design. Furthermore, potentially important clinical parameters, such as initial clinical tumor stage, grade, and time from diagnosis to RNU, were not available for analysis. Time from ureteroscopic ablation to RNU was not available for analysis but may have had a significant impact on final pathologic stage and oncologic outcome after RNU. Thus, only well-designed prospective studies can confirm that delay of RNU because of initial ureteroscopic ablation does not compromise the oncologic outcomes of UTUC patients. With the rarity of the disease, however, a retrospective study design, from multiple high-volume cancer centers, may provide the best possible clinical data.

Retrospective analysis of one of the largest international cohorts of patients who were treated with RNU for UTUC suggests that the initial attempt at endoscopic tumor ablation is not associated with inferior oncologic outcomes after RNU. Our data support the continued role of ureteroscopic tumor ablation in selected patients with UTUC. Prospective studies are needed to confirm our findings.

\section{Acknowledgment}

We want to thank members from the Upper Tract Urothelial Carcinoma Collaborative Group who participated in this study: Wareef Kabbani, UT Southwestern Medical

Table 2. Multivariate Cox Regression Analysis for Prediction of Disease Recurrence and Cancer-Specific Mortality After Radical Nephroureterectomy

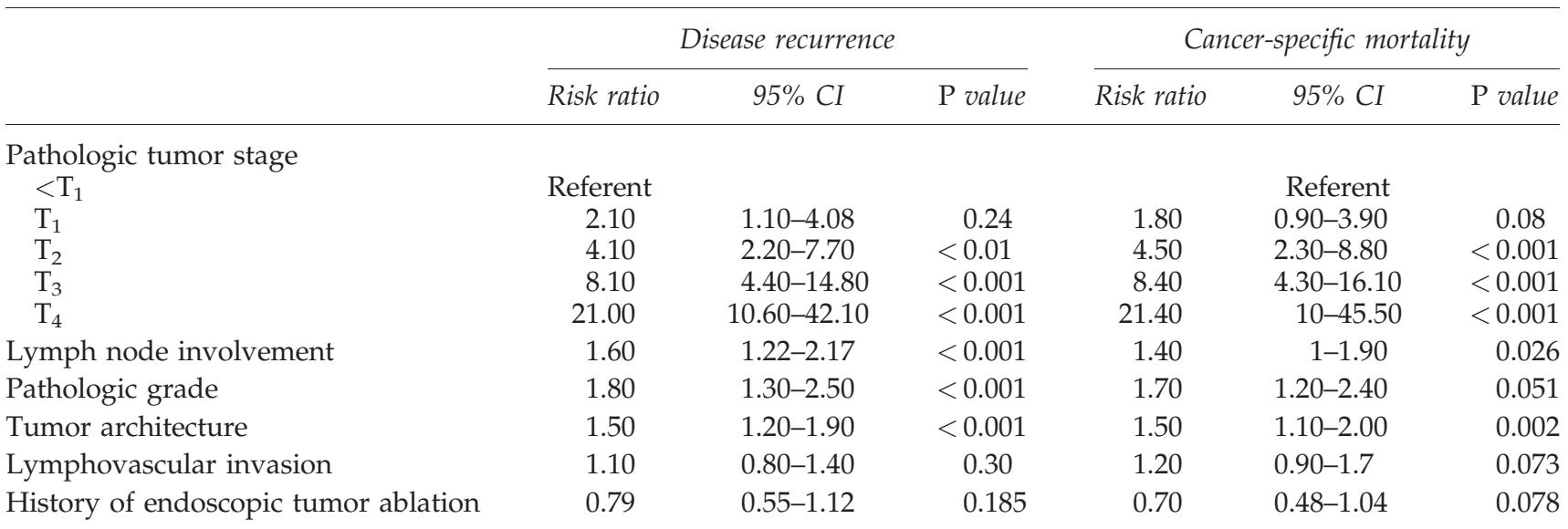

$\mathrm{CI}=$ confidence interval. 
Center, Dallas, Texas; Charles C. Guo, UT M.D. Anderson Cancer Center, Houston, TX; Roberto Bertini, Vita-Salute University, Milan, Italy; Andrea Haitel Medical University of Vienna, Austria; Jeffrey Wheat, J. Stuart Wolf, Jr, University of Michigan, Ann Arbor, Michigan; Casey K. Ng, Douglas S. Scherr, Yingbei Chen, Cornell University, New York, NY; Philipp Stroebel, Maurice Stephan Michel, Universitätsklinikum Mannheim, Mannheim, Germany; Shuji Mikami, Takeo Kosaka, Masaru Isida, Keio University School of Medicine, Tokyo, Japan; Mario A. Fernández, Clínica Alemana de Santiago, Santiago, Chile.

\section{Disclosure Statement}

No competing financial interests exist.

\section{References}

1. Raman JD, Scherr DS. Management of patients with upper urinary tract transitional cell carcinoma. Nat Clin Pract Urol 2007;4:432-443.

2. Zigeuner R, Pummer K. Urothelial carcinoma of the upper urinary tract: Surgical approach and prognostic factors. Eur Urol 2008;53:720-731.

3. Tomera KM, Leary FJ, Zincke H. Pyeloscopy in urothelial tumors. J Urol 1982;127:1088-1089.

4. Kulp DA, Bagley DH. Does flexible ureteropyeloscopy promote local recurrence of transitional cell carcinoma? I Endourol 1994;8:111-113.

5. Boorjian $\mathrm{S}, \mathrm{Ng} \mathrm{C}$, Munver $\mathrm{R}$, et al. Impact of delay to nephroureterectomy for patients undergoing ureteroscopic biopsy and laser tumor ablation of upper tract transitional cell carcinoma. Urology 2005;66:283-287.

6. Chang SS, Hassan JM, Cookson MS, et al. Delaying radical cystectomy for muscle invasive bladder cancer results in worse pathological stage. J Urol 2003;170:1085-1087.

7. Herr HW, Sogani PC. Does early cystectomy improve the survival of patients with high risk superficial bladder tumors? J Urol 2001;166:1296-1299.

8. Roscigno M, Shariat SF, Freschi M, et al. Assessment of the minimum number of lymph nodes needed to detect lymph node invasion at radical nephroureterectomy in patients with upper tract urothelial cancer. Urology 2009; 74:1070-1074.

9. Gerber GS, Lyon ES. Endourological management of upper tract urothelial tumors. J Urol 1993;150:2-7.
10. Grossman HB, Schwartz SL, Konnak JW. Ureteroscopic treatment of urothelial carcinoma of the ureter and renal pelvis. J Urol 1992;148:275-277.

11. Greene FL, Page DL, Fleming ID, et al. American Joint Committee on Cancer (AJCC) Staging Manual. 6th ed. Philadelphia: Springer; 2002.

12. Epstein JI, Amin MB, Reuter VR, Mostofi FK. The World Health Organization/International Society of Urological Pathology consensus classification of urothelial (transitional cell) neoplasms of the urinary bladder. Bladder Consensus Conference Committee. Am J Surg Pathol 1998;22:1435-1448.

13. Hall MC, Womack S, Sagalowsky AI, et al. Prognostic factors, recurrence, and survival in transitional cell carcinoma of the upper urinary tract: A 30-year experience in 252 patients. Urology 1998;52:594-601.

14. Keeley FX, Kulp DA, Bibbo M, et al. Diagnostic accuracy of ureteroscopic biopsy in upper tract transitional cell carcinoma. J Urol 1997;157:33-37.

15. Brown GA, Matin SF, Busby JE, et al. Ability of clinical grade to predict final pathologic stage in upper urinary tract transitional cell carcinoma: Implications for therapy. Urology 2007;70:252-256.

16. Margulis V, Youssef RF, Lotan Y, et al; Upper Tract Urothelial Carcinoma Collaborative Group. Preoperative multivariable prognostic model for prediction of nonorgan confined urothelial carcinoma of the upper urinary tract. I Urol 2010;184:453-458.

Address correspondence to: Vitaly Margulis, M.D.

Department of Urology

The University of Texas Southwestern Medical Center 5323 Harry Hines Boulevard Dallas, TX 75390-9110

E-mail: vitaly.margulis@utsouthwestern.edu

$$
\begin{aligned}
& \text { Abbreviations Used } \\
\mathrm{CSS} & =\text { cancer-specific survival } \\
\mathrm{CT} & =\text { computed tomography } \\
\mathrm{DFS} & =\text { disease-free survival } \\
\mathrm{IVU} & =\text { intravenous urography } \\
\mathrm{MRI} & =\text { magnetic resonance imaging } \\
\mathrm{RNU} & =\text { radical nephrouretectomy } \\
\mathrm{UTUC} & =\text { upper urinary tract urothelial carcinoma }
\end{aligned}
$$



This article has been cited by:

1. Bassel G Bachir, Wassim Kassouf. 2012. Efficacy of instillations with chemotherapy or immunotherapy following endoscopic resection for upper tract urothelial carcinoma. Expert Review of Anticancer Therapy 12:1, 63-75. [CrossRef] 